\title{
Fatigue versus disengagement in unilateral neglect
}

\author{
Ian H Robertson, $\mathbf{N}$ North
}

\begin{abstract}
A letter cancellation task revealed neglect of the lower left quadrant when cancellation began at the top, but of the upper left quadrant when it began in the bottom. When the cancellation was done line by line through a "window" which covered all but the line currently worked on, the quadrant effects disappeared, so that fatigue could not explain these effects. An alternative hypothesis is that as the cancellation progressed, the subject's attention was attracted by progressively more stimuli on the right, and that disengagement difficulty and hence neglect increased proportionately to the number of stimuli.
\end{abstract}

$(\Im$ Neurol Neurosurg Psychiatry 1993;56:717-719)

Several studies have suggested that neglect need not only occur in the left-right dimension, but also vertically ${ }^{1-3}$ and radially ${ }^{4-6}$ suggesting that attention in space is guided by multidirectional and interacting attentional systems.

Fatigue is known to exacerbate unilateral neglect $^{7}$ and one study ${ }^{8}$ has investigated whether the raised incidence of neglect of the lower left quadrant in cancellation tasks ${ }^{5}$ was attributable to fatigue. This study found that in three out of five cases, neglect persisted in being more prevalent in the lower left quadrant, even when the patients began cancellation at the bottom. In the other two cases, however, neglect appeared in the top left quadrant with bottom-start, suggesting that in these cases at least, fatigue may have accounted for the quadrant effect, with attention "dropping off" as the task progressed.

This study attempts to clarify this phenomenon in a patient with unilateral neglect who showed marked left neglect, particularly of the lower left quadrant in a cancellation task.

\section{MRC Applied}

Psychology Unit, 15 Chaucer Road, Cambridge, UK

I H Robertson

Psychology

Department, Odstock

Hospital, Salisbury,

UK

N North

Correspondence to: Dr Robertson

Received 8 June 1992 and in final revised form

13 October 1992.

Accepted 16 October 1992 territory of the right middle cerebral artery.

The subject made a slow recovery of function until he suffered an extension to his stroke on 29 April 1991, the left sided weakness increased, and his left pupil was more dilated than his right. A CT scan reported after this extension that there was evidence of further infarction, and that this included the area of the anterior cerebral artery superiorly (slices 9, 10 and 11). The CT scan showed no focal abnormality in the left hemisphere, though several well defined areas of gliosis were present near the frontal horn of the right lateral ventricle. The latter also showed increased dilation compatible with a degree of local atrophy in that area.

Neuropsychological assessment revealed severe left visual neglect, as measured by the "conventional" items of the Behavioural Inattention Test $^{9}$ (namely letter cancellation, star cancellation, line bisection, figure copying, drawing from memory and line bisection), on which he scored in the severe neglect range (54 out of a maximum of 146). The left omissions were very unlikely to have been attributable to his hemianopia because he showed a very similar pattern of omissions during blindfolded tactile search of the star cancellation test of the Behavioural Inattention Test: this was done by sticking small raised circles of paper on the target stars and requiring him to search for them with his fingers. On this tactile version of the test, he omitted $27 / 56$ of the targets, compared with $24 / 56$ omissions on the visual version of the test.

His immediate Logical Memory (Revised Wechsler Memory Scale), was on the 57th percentile and delayed memory at the 64th percentile, and he showed no significant perseveration on the Nelson version of the Wisconsin Card Sorting Test (where the cards were aligned vertically rather than horizontally to minimise the effects of neglect: total errors: 11/48; number of perseverative errors: 4/11). He scored in the normal range on the Similarities and Comprehension subtests of the Wechsler Adult Intelligence Scale (Revised), with scores on the 38 and 75 percentile. His Digit Span was also normal (90 percentile). He was, however, considerably impaired on Object Assembly and Block Design. ( $<1$ and 10 percentile, respectively). His estimated premorbid IQ was 110 (National Adult Reading Test) and he was not significantly depressed nor anxious (Hospital Anxiety and Depression Scale-4 and 0 for depression and anxiety respective- 
ly). He was fully oriented for time, place and person.

\section{Experiment 1}

On the letter cancellation (" $E$ " and " $R$ ") subtest of the Behavioural Inattention Test $^{9}$ the subject showed very marked neglect, particularly of the left lower quadrant, when the subject began at the top-leftmost target. When the subject was instructed to begin at the bottom (without instruction about which side of the bottom line to start on) the prevalent omissions were in the top left, and he began at the far left side of the bottom line on $9 / 10$ occasions. A total of 20 administrations of this task were given, half with a top start, and half with a bottom start, with the two conditions randomised across the 20 occasions.

The probability of a particular target letter on each of the five rows of letters being omitted was calculated over the ten administrations of each condition. Mean probabilities of omissions for the four quadrants were calculated (excluding the middle row of the five row cancellation test). The table shows the 16 probabilities, including the probabilities of omissions for the left and right sides of the top and bottom lines respectively.

The probabilities of omissions in the left lower and upper quadrants reversed from the top start to the bottom start. With a top start, the subject was much more likely to omit targets in the bottom left of the test, and with a bottom start, to omit them in the top left of the test. This would appear to support Mark and Heilman's ${ }^{8}$ notion of a fatigue effect, so that neglect increases as the subject progresses in the task, due to decreased arousal.

\section{Experiment 2}

If fatigue underlies the phenomenon reported above, then the same cancellation task performed line by line through a "slit" which allows the subject to see only one line at a time should produce the same effect as in the previous experiment. If, on the other hand, the altitudinal effects of Experiment 1 were simply caused by a progressive increase in overall ambient stimulus input as the subject progressed through the test, then one should expect to see no increase in neglect between the first and last lines cancelled. In piloting this procedure with the cancellation task used in Experiment 1, errors declined to almost zero: that is, when the subject completed the cancellation task line by line through a slit in a piece of paper, which moved down one line at a time as the subject announced completion of a line, then the number of omissions

Table Probability of omissions by quadrant and by top and bottom lines

\begin{tabular}{llllll}
\hline & \multicolumn{3}{l}{ Left side } & & \multicolumn{2}{l}{ Right side } \\
\cline { 2 - 3 } \cline { 5 - 6 } & $\begin{array}{l}\text { Top } \\
\text { start }\end{array}$ & $\begin{array}{l}\text { Bottom } \\
\text { start }\end{array}$ & $\begin{array}{l}\text { Top } \\
\text { start }\end{array}$ & $\begin{array}{l}\text { Bottom } \\
\text { start }\end{array}$ \\
\hline Top 2 lines & 0.25 & 0.54 & & 0.18 & 0.32 \\
Top line only & 0.05 & 0.60 & & 0.00 & 0.47 \\
Bottom 2 lines & 0.39 & 0.11 & & 0.14 & 0.01 \\
Bottom line only & 0.45 & 0.07 & & 0.14 & 0.00 \\
\hline
\end{tabular}

decreased to less than $10 \%$. A more difficult cancellation task was therefore used, which yielded omissions even when performed through the "slit".

The test was a reduced version of the " $\mathrm{d} 2$ " cancellation test. ${ }^{10}$ This consisted of 6 rows, each of 47 letters. The letters are all "p" or "d" and in addition, each letter has one or two lines above or below it, for example:

$$
\mathrm{d}, \mathrm{p}, \mathrm{d}_{\|}, \mathrm{p}_{1}, \mathrm{~d}_{1}, \mathrm{p}, \mathrm{d}, \mathrm{p}, \mathrm{d}, \mathrm{p} \text {. }
$$

The task is to cancel only those d's which have two lines, either two above, two below, or one above and one below. Neglect is known to worsen as the complexity of a task increases, ${ }^{11}$ and thus it was not surprising to find that errors were considerable when this task was carried out line by line through a window in the manner described above.

More specifically, the subject was told to cancel out all the target d's which had two lines associated with them. In the top-down procedure, the subject was presented with the first line through a $1 \mathrm{~cm}$ wide slit in an A4 width sheet of paper, the slit being the same length as each row, namely $22 \mathrm{~cm}$. The subject was told to say when he thought he had finished a particular line and then the slit was moved down. The test was repeated fifteen times, five with no instructions and normal presentation (that is, no slit), five with slit and top start, and five with slit and bottom start. These three conditions were randomised across trials. Without a window, the subject started on the top line and progressed systematically downwards, and hence this condition was always in effect a "top start".

In each version of the test, the subject omitted many more targets on the left than on the right (166 left $v 66$ right in free vision).

On the top line in free vision he missed several of the leftmost targets in four out of five trials, a not surprising finding given the much greater complexity of the $\mathrm{d} 2$ test than the previous cancellation test. This was also true for the top line after a top start, where he missed some leftmost targets in 4 out of 5 trials. After a bottom start, he missed the leftmost targets on the bottom line in 3 out of 5 trials.

In free vision, the subject missed many more targets on the bottom two lines than in the top two lines (139 in the bottom two versus 93 in the top two; $t=-2 \cdot 2 ; \mathrm{p}<0 \cdot 05)$. In both the window conditions, however, the difference between top and bottom errors were negligible (67 errors top and 66 errors bottom after top start; 66 errors top and 59 errors bottom after bottom start).

\section{Discussion}

The critical results in Experiment 2 are not the lack of difference between a top and bottom start, but rather the lack of difference between top and bottom line errors within each of these conditions. The bottom start condition is perhaps better seen simply as a replication of the top start condition.

The $\mathrm{d} 2$ test yielded considerable left sided 
errors, even in the starting line, whether that was the top or bottom. It is therefore difficult to argue that the effect of the window was simply to reduce ambient stimulus density so as to make the task too easy, and hence make a progressive fatigue-induced increase in errors as the test progressed unlikely.

When the $\mathrm{d} 2$ test was given without the window, the previously observed pattern of results persisted, namely that the bottom left quadrant showed more omissions than other quadrants. That this could not be attributed to altitudinal neglect was shown by the reversal condition in Experiment 1. The disengagement and fatigue accounts therefore remain as possible hypotheses, each having quite different implications for, among other things, the nature of rehabilitation of unilateral neglect.

The window in Experiment 2 did not eliminate neglect, even in the first line cancelled, yet there was no increase in neglect as the test progressed, as had been observed with free vision. Hence time-on-task itself could not explain the findings, but only time-on-task in conjunction with progressively enlarging ambient stimulation. Had fatigue been an important factor, Experiment 2 should have revealed a progressive increase in errors as the window-controlled cancellation proceeded, given that the task was still very difficult and errorful for the subject, even in the first row. Given this argument, what then might underlie the findings of Experiment 1, namely that a top start revealed bottom left neglect, and a bottom start produced top left neglect?

One possible explanation draws on Posner's ${ }^{12}$ theory of unilateral neglect. According to this theory, neglect is attributable to a disorder in disengaging attention from stimuli on the side ipsilateral to the lesion.

Applying this to the current data, the subject begins cancellation on the first line (top or bottom), and he attends to the targets there, including those on the right. At this stage, there are, say, $x$ stimuli from which he has to disengage attention to progress to the left side of the second line. Once attending to the second line, there are now $2 x$ stimuli from which attention must be disengaged (assuming that the fact of having attended to the right stimuli on the first line leaves them to some extent still active in holding attention there). Cancelling the third, fourth and fifth lines involves the action of $3 x, 4 x$ and $5 x$ stimuli respectively on the impaired attentional disengagement system.

In summary, the present pattern of results are explained by a hypothesis which argues that the more stimuli there are over which attention is deployed, the greater the difficulty the person will have in disengaging that attention to the left side of any display. Mark et $a l^{13}$ compared cancellation under two different conditions, one a standard procedure where subjects made a mark on each target, and the other where cancellation consisted of removing each cancelled stimulus from the array, that is, each cancellation reduced the total number of stimuli in the visual field. This latter procedure produced significantly less neglect than the standard one, and this finding is compatible with the disengagement hypothesis.

Such an explanation may have important therapeutic implications. The prevalent form of therapy for unilateral neglect involves training patients to scan into their neglected field. ${ }^{14}$ Strategies which focus more specifically on helping patients disengage their attention from objects and events on their non-neglected side may prove to be more effective.

The present results do not, however, explain the results of Mark and Heilman who found that neglect of the left lower quadrant during cancellation persisted even after the subjects began at the bottom of the task. What this confirms is that neglect is not a unitary phenomenon, and that a particular manifestation, for example, quadrant neglect, may result from different underlying disorders. The three cases reported by Heilman may well be true examples of altitudinal neglect. ${ }^{1-3}$

1 Shelton PA, Bowers D, Heilman KM. Peripersonal and vertical neglect. Brain 1990;113:191-205.

2 Rapscak SZ, Cimino CR, Heilman KM. Altitudinal neglect. Neurology 1988;38:277-81.

3 Bender MB, Teuber HL. Spatial organisation of visual perception following injury to the brain. Arch Neurol Psychiatry 1948;59:39-62.

4 Morris R, Mickel S, Brooks M, Swavely S, Heilman KM. Recovery from neglect. 7 Clin Exper Neuropsychol 1985; 7:609.

5 Halligan PW, Marshall JC. Is neglect only lateral? A quadrant analysis of line cancellation. $\mathcal{f}$ Clin Exper Neuropsychol 1989;11:793-8.

6 Mennemeier M, Wertman E, Heilman KM. Neglect of near peripersonal space. Brain 1992;115:37-50

7 Fleet WS, Heilman, KM.. The fatigue effect in unilateral neglect. Neurology 1986;36(Suppl 1):258.

8 Mark VW, Heilman KM Does fatigue account for left peripersonal neglect? $f$ Clin Exper Neuropsychol 1988; 10:335.

9 Wilson B, Cockburn J, Halligan P. Behavioural Inattention Test. Fareham, Hants; Thames Valley Test Company, 1988.

10 Brickenkamp R. (1962) Test d2. Zurich: Verlag fur Psychologie, Dr CJ Hogrefe.

11 Rapcsack SZ, Verfaellie M, Fleet S, Heilman KM. Selective attention in hemispatial neglect. Arch Neurol 1989;46:172-8.

12 Posner MI, Cohen Y, Rafal RD. Neural systems control of spatial orienting. Proceedings of the Royal Society of London 1982; B, 298:187-98.

13 Mark VW, Kooistra CA, Heilman KM. Hemispatial neglect affected by non-neglected stimuli. Neurology 1988;38:1207-11

14 Weinberg J, Diller L, Gordon W, et al. Visual scanning training effect on reading-related tasks in acquired right brain damage. Arch Physical Med Rehab 1977;58: 479-86.

15 Weinberg M, Diller L, Gordon W, et al. Training sensory awareness and spatial organisation in people with right brain damage. Arch Physical Med Rehab 1979;60:491-6. 